\title{
Flow calculations in intake structures of hydropower plants
}

\author{
Alexander Gazaryan ${ }^{1 *}$, and Oleg Glovatskii ${ }^{1,2}$ \\ ${ }^{1}$ Research Institute of Irrigation and Water Problems, Tashkent, Uzbekistan \\ ${ }^{2}$ Tashkent Institute of Irrigation and Agricultural Mechanization Engineers, Tashkent, Uzbekistan
}

\begin{abstract}
Research is carried out for design institutes in which the structures of pumping stations are designed. In the regional conditions of the Republic of Uzbekistan, when operating pumping stations, the problem of protecting equipment from pumps and fin is of particular importance, as well as the stability of water supply structures and the creation of favorable hydraulic conditions for supplying flow to the pumps. The main factors in the flow conditions are the calculations associated with suspended particles. The purpose of the research was to study the kinematics of the flow of intake structures in the problem of protecting equipment from pumps and fin. The research subject was to identify the regularities of the operation of intake structures depending on the structure of the flow supply. Experimental methods of hydraulic research of intake structures of pumping stations are used in the work. The analysis of the current state of water supply to the intake structures of pumping stations is carried out based on calculation schemes, which make it possible to establish the nature of the influence of flow parameters and soil particles on this process.
\end{abstract}

\section{Introduction}

Based on the operating conditions of pumping stations (PS), scientific research is being carried out in the world to improve their designs and ensure the efficiency of their operation. The Hydroelectric stations (HES) are a responsible complex. Their designing and usage require the acquaintance with broad around of hydromechanical discipline. The scheme of the count of the energy potential HES in a certain fold with consumption Q and pressure $\mathrm{H}$ must consider that part of pressure gets lost when a motion of water from the fence of water before unit [1-7].

In this direction, ensuring a normal water level in intake structures to prevent the operation of pumping units in the cavitation mode is of particular relevance.

Scientific research on intake structures is carried out in the fundamental research of the authors to study the problems of improving the theory of unsteady water movement in the "channel - pumping station" system to improve their interconnections [1-4]. It is the

\footnotetext{
* Corresponding author: gazaryan.lex@gmail.com
} 
investigation of the laws of change in the kinematic characteristics of the flow in the intake structures of large pumping stations, mainly of the chamber and block type, and assessing the influence of the design of the intake structures on the hydraulic conditions for supplying water to the pumps, in particular, extreme ones, and on the operational parameters of the pumps during unsteady processes [8-18].

\section{Methods}

When performing the work, methods of hydraulic study of the flow structure based on wellknown measurement methods were used. The reliability of the data obtained in the course of theoretical research is proved by mathematical methods of checking the adequacy of experimental results and field studies of a quantitative assessment of the conditions of water intake from the middle and extreme water intake chambers and objective data of these conditions on the reliability and efficiency of operation of the pumps $[5,6]$.

\section{Results and Discussion}

By intake structures, we mean structures that ensure the functioning of the main pumping equipment at the boundary sections of the flow before and after building the PS and HES. There are many descriptions of design schemes that allow us to establish the nature of the influence of flow parameters in these sections and suspended foreign particles (sediment) on this process (figure 1).

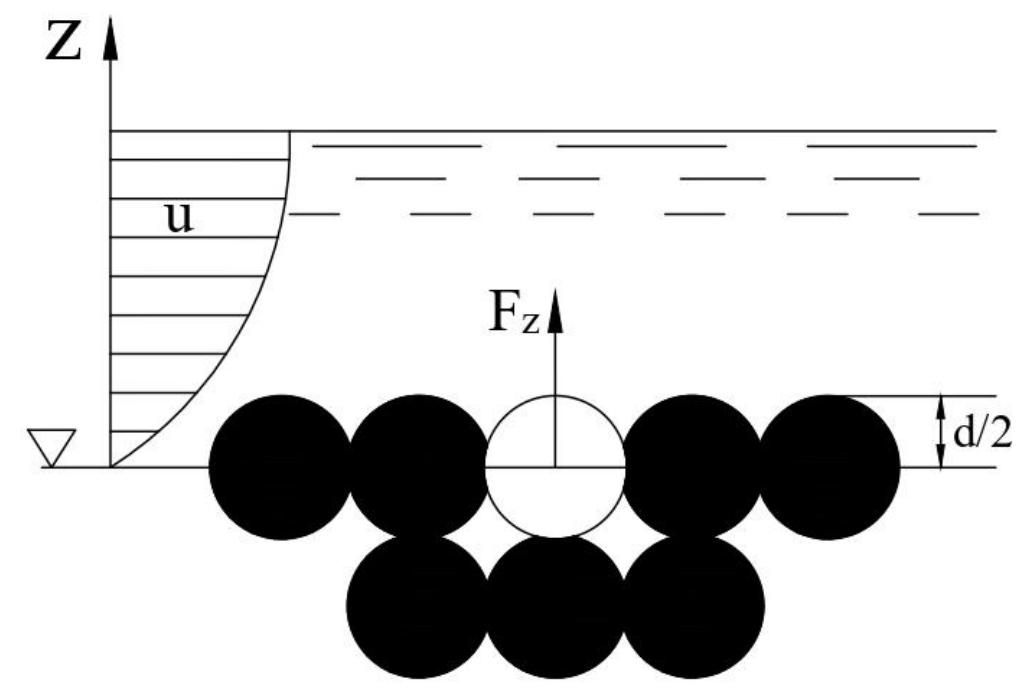

Fig. 1. The scheme of weighing particles of bottom soil

However, to this day, the question remains unanswered whether the weighing of soil particles is directly related to their flow over a time-averaged flow and the action of vertical turbulent velocity pulsations or the attracting force arising between a moving stream and a fixed bottom. The proposed schemes are not brought to a complete calculation of the weighing conditions and usually end with criteria-type relations [2, 7].

The dynamic effect of the flow on the particle of the bottom soil determines the separation of the particle from the system of surrounding particles and its weighing by the flow. Currently, it is believed that the action of the following forces determines the 
weighing of particles of bottom soil [19-30]:

- Gravity

$$
F_{G}=a_{G}\left(\frac{\rho_{\tau}}{\rho}-1\right) \frac{\pi d^{3}}{6}
$$

- $\quad$ frontal pressure forces

$$
F_{x}=\rho a_{x} C_{D} \frac{\pi d^{2}}{4}-\frac{u_{k}^{2}}{2}
$$

- $\quad$ lifting power

$$
F_{z}=\rho a_{я} C_{n} \frac{\pi d^{2}}{4} \frac{u_{k}^{2}}{2}
$$

- $\quad$ adhesion forces

$$
F_{\sigma}=\sigma_{p} \frac{\pi d^{2}}{4}
$$

- $\quad$ hydrostatic loading forces

$$
F_{p}=\rho g h a_{p} \frac{\pi d^{2}}{4}
$$

Here $a_{G}, a_{x}, a_{z}$ are the coefficients of the form of soil particles with a diameter $d ; a_{p}$ is coefficient taking into account the area over which unbalanced hydrostatic pressure acts; $C_{D}$ is coefficient of hydrodynamic resistance; $C_{\Pi}$ is the coefficient of lifting force; $\sigma_{p}$ is soil resistivity to rupture (referred to the unit area of the midsection of the particle); $u_{\kappa}$ is current (instantaneous) speed at the level of the peaks of the roughness protrusions.

Let us consider in more detail each of the active forces.

Gravity $F_{\mathrm{G}}$ can be calculated correctly if the particle volume and density are known. Since the real particle, as already noted, differs in the shape of the ball, its real volume $V_{\mathrm{p}}$ will differ from the volume of the ball $V_{\mathrm{b}}$ with the same size

$$
\frac{V_{p}}{V_{b}}=a_{G}
$$

Considering the forces acting on the particle, we note that the frontal pressure force can cause the rolling of single particles lying on a smooth surface or significantly protruding from the layer of surrounding particles; however, in this case, the horizontal directional frontal pressure force cannot cause the particle to weigh.

In a system of equal-sized particles, the force of frontal pressure $F_{\mathrm{x}}$ will be balanced by the reaction of the surrounding soil grains. Therefore, the only active force that determines 
the separation of a particle under these conditions is the lifting force $F_{\mathrm{z}}$.

The lifting force is determined by the highest actual velocity of flow around the particle $u_{\kappa}$ (velocity at the height of the roughness protrusions), the nature of the pressure distribution over its surface, the shape of the particle, and the lifting force coefficient $\mathrm{C}_{\mathrm{n}}$.

Among the forces holding the particle in the system, in addition to gravity, are adhesion forces. Cohesive forces act both in cohesive and dispersed soils, determining the mutual attraction of particles to each other. The adhesion forces of fine-grained dispersed soils and their effect on the erosion resistance of particles are considered earlier [8].

The hydrostatic pressure acting on a particle will not affect its stability only if the pressure is applied to all points of its surface and, thus, is balanced. This happens when the particle is surrounded by a layer of water of considerable thickness. However, there are suggestions that particles of bottom soil are affected by hydrostatic loading, increasing with depth [2].

To illustrate, we consider the effect of hydrostatic pressure forces on a rectangular particle with a "contact spot" with an area of He on the lower face. The hydrostatic pressure forces acting along the lateral faces of the particle are balanced and do not affect its stability. Under the conditions of a free particle, the force $\rho g h d^{2}$ acts on its lower face, and the force $(h-d) d^{2} \rho g$ acts on the upper face. The difference between these forces determines the strength of buoyancy (figure 2.). If the particle on the lower face has a "contact spot" that does not transmit hydrostatic pressure, then the force $p g h\left(d^{2}-\omega\right)$ will act on the lower face (minus the area of the "contact spot"), and the buoyancy force will be equal

$$
\operatorname{pgh}\left(d^{2}-\omega\right)-p g(h-d) d^{2}
$$

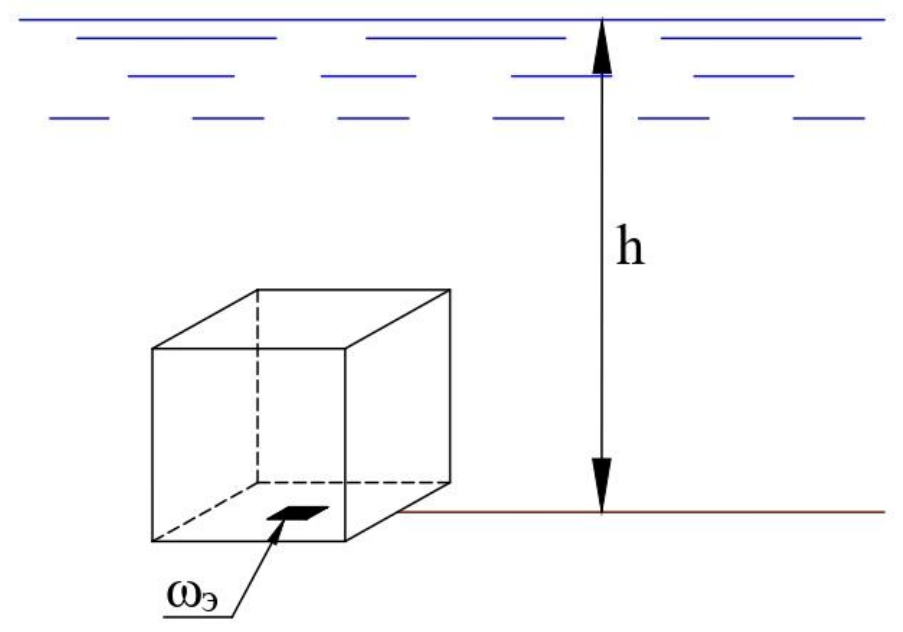

Fig. 2. Scheme for calculating hydrostatic loading

The resulting vertical force acting on the particle in the liquid at rest will be determined by the difference between the particle weight (in the air) $p_{\mathrm{r}} g d^{3}$ and the buoyancy force. This force is greater than the force of gravity acting on a particle in water

$$
\left(p_{\mathrm{T}}-p\right) g d^{3}+p g h \omega .
$$


The second term in this expression is the force of the hydraulic load acting on the particle. Its value is determined by the area of the "contact spot" $\omega$ and the depth of immersion of the particle under level $h$. The above data on the thickness of the water layer make it possible to estimate the hydrostatic loading with the loading force $\Pi=p g h \omega$ and the force of gravity acting on the particle in the water $\mathrm{G}=\left(p_{\mathrm{T}}-p\right) g d^{3}$.

The calculation according to this ratio at $h=1 \mathrm{~m}$ for particles of various sizes shows that the ratio of hydrostatic loading to the force of gravity acting on the particle increases with decreasing particle diameter (table.).

Table - The ratio of hydrostatic loading to particle weight

\begin{tabular}{|c|c|c|c|c|c|}
\hline $\mathrm{d} \mathrm{mm}$ & 1.0 & 0.3 & 0.1 & 0.03 & 0.01 \\
\hline $\mathrm{P} / \mathrm{G}$ & $5 \cdot 10-7$ & $1.6 \cdot 10-5$ & $5 \cdot 10-4$ & $1.6 \cdot 10-2$ & $5 \cdot 10-1$ \\
\hline
\end{tabular}

The calculation data show that for full-scale watercourses, the hydrostatic loading for particles $d>0.03 \mathrm{~mm}$ is small compared to the force of gravity acting on the particle. It may not be taken into account in calculations of soil resistance to erosion. This conclusion is consistent with the measurement data of C. E. Mirtskhulava and the authors of $[8,9]$.

Let us consider in more detail the factors determining the lifting force $F_{\mathrm{z}}$. The value of this force depends on the square of the actual speed at the top of the protrusion of the roughness $u_{\mathrm{r}}$. Therefore, the success of the quantitative determination of the particle weighing conditions largely depends on the accuracy of the speed calculation. The speed is determined differently depending on the resistance mode. The resistance mode of the river flow is known to be determined by the parameter $u k_{\mathrm{s}} / v$. In the absence of bottom channel forms, the value of $k_{\mathrm{s}}$ is close to $d$, and in this case, the parameter $u k_{s} / v$ determines the regime of flow around the soil grains [31-35].

In the presence of bottom channel forms, the channel resistance increases sharply. The equivalent roughness $k_{s}$ determined by the resistance significantly (sometimes by several orders of magnitude) exceeds the value $d$. Therefore, at high values of $u k_{s} / v$, the parameter $u d / v$ may remain small. In quadratic resistance mode with $u d / v>50$

$$
\mathrm{u}_{\mathrm{k}} / \mathrm{u}=6.75 \text {. }
$$

The vertices of particles oriented vertically with the average diameter (at $z_{\kappa}=\frac{d}{2}$ ) will flow around at a lower speed equal to $6.75 u$.

Initial weighing of protruding particles will occur during periods of action of the largest longitudinal velocity pulsations.

The measurement data show that the standard of longitudinal ripples near the wall is close to an average of $2.7 u$, while the largest ripple is $8.1 \mathrm{u}$, which is close to the average velocity.

The data obtained show that the maximum recorded speeds when cleaning from bottom sediments near the bottom are on average 1.5 times higher than the average value of the rate during siltation $[5,8]$.

The calculation of the free cross-sections in the flowing part of the pumps according to the existing methods is determined by the high-speed pressure calculated from the average speed and does not consider the changes in the velocity diagram when changing the pump modes. The proposed new elements eliminate the disadvantages of these techniques [1013]. The authors have created new pump designs with reduced wear and increased ability to control the modes [14-17]. Vortex zones are formed at the walls in the spiral chamber, in 
the suction nozzle, and the discharge nozzle, and when installing the separating spiral planes, the transit flow speed increases, and, accordingly, the vortex zones are practically eliminated. At values $\sigma_{\mathrm{y}}<\sigma_{\mathrm{\kappa p}}$, the vortex bundle breaks up into smaller ones, which leads to a change in the period and shape of the pressure pulsation. The range of pulsations at values $\sigma_{\text {y of y close to }} \sigma_{\text {y increases and then decreases. }}$

For high-frequency pulsation, the process proceeds somewhat differently. The main difference lies in the fact that the pressure pulsation frequency decreases near the cavitation stall, and the swing increases (figure $3 \mathrm{~b}$ ).

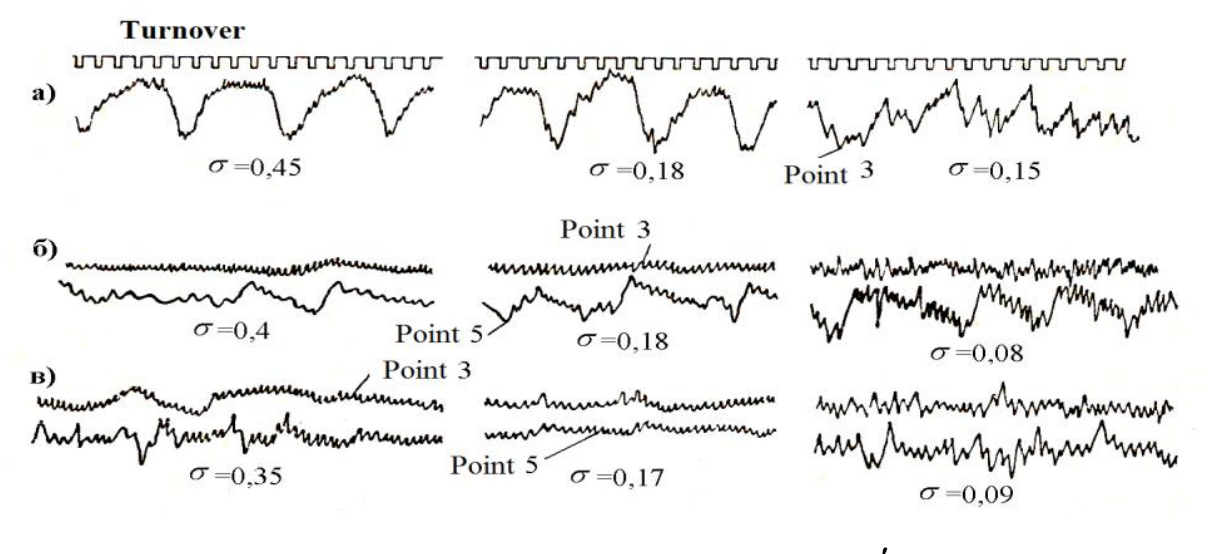

Fig. 3. Combined oscillograms of pressure pulsations: a) - $a_{0}=14 \mathrm{~mm}, n_{1}=110 \mathrm{r} / \mathrm{m}$; б) $-\mathrm{a}_{\mathrm{o}}=18 \mathrm{~mm}$, $\left.n_{1}^{\prime}=90 \mathrm{r} / \mathrm{m} ; \mathrm{B}\right)-\mathrm{a}_{\mathrm{o}}=22 \mathrm{~mm}, n_{1}^{\prime}=80 \mathrm{r} / \mathrm{m}$.

\section{Conclusions}

1. Studies carried out at RIWP with the participation of the authors have shown that the obtained ratios make it possible not only to use scientifically grounded methods for calculating the known intake structures of the PS (front chambers, water intakes), but also to analyze the design schemes of dynamic and kinematic flow supply conditions, which allow to establish the nature of the influence of flow parameters and soil particles of bottom and suspended sediments on the process of their layout [18,19].

2. For natural watercourses of the intake structures of the PS and HES, the hydrostatic surcharge for particles $\mathrm{d}>0.03 \mathrm{~mm}$ turned out to be small in comparison with the gravity acting on the particle and may not be taken into account in the calculations of soil resistance to erosion. The data obtained show that the maximum recorded velocities when cleaning from bottom sediments, on average, are 1.5 times higher than the average value of the velocity during silting.

3. Recommendations for selecting rational designs and operating modes of intake structures and main equipment of the PS allow making technical and economic comparisons of options at the design stage of pumping stations and take into account during operation.

4. The developed method for calculating the flow paths of pumps based on applying the formulated methods of applying the theory of hydrodynamics makes it possible to design PS and HES with optimal characteristics [20,21]. The method allows for the modernization of the flow parts of hydrounits of various types and different parameters. 


\section{References}

1. Glovatsky O.Ya., Nasyrova N.R., Artikbekova F.K. Some aspects of the use of pumping stations in the development of the water-resource sphere of Central Asia in the XXI century // Collection of reports of the XX International scientific-practical conference "Northern Sea Route, water and land transport corridors as the basis for the development of Siberia and the Arctic in the XXI century." Volume I. - Tyumen: TIU, pp.69-74. (2018)

2. Glovatsky O. Ya., Nasyrova N. R., Saparov A. B., Berdaliev M. K. Some methods for calculating the unsteady movement of water in the "channel - pumping station" system // Scientific and Practical Journal "Ways to Improve Irrigation Efficiency agriculture"Novocherkassk, No. 1 (73),-- pp. 160-165, (2019)

3. A.Krutov Optimal location of an intake at a reservoir prone to salt diffusion (FORM2020 (XXIII International Scientific Conference "Construction the Formation of Living Environment 2020": September 23-26, 2020 in Hanoi (Vietnam)) Anatoly Krutov et al 2020 IOP Conf. Ser.: Mater. Sci. Eng. 869072020.

4. O.R. Azizov, A.S. Gazaryan, N.R. Nasyrova, N. M. Ismailov Improving the safety of mating structures of pumping stations with transients // Scientific and practical journal "Ways to improve the efficiency of irrigated agriculture" - Novocherkassk, No. 3 (75), pp 74-78, (2019)

5. A. Yangiev, K. Salyamova, K. Turdikulov and X. Fayziev Dynamics of an earth dam with account for rheological properties of soil under dynamic effect.IOP Conf. Series: Materials Science and Engineering 869 (2020) 072005.

6. Rustamov Sh. R., Nasirova N. R. Constructive peculiarities of modernized centrifugal pump // European science review, № 3-4, Vienna. pp-278-280, (2018)

7. Barton A F, Mala-Jetmarova H. An Algorithm for Minimization of Pumping Costs in Water Distribution Systems using a Novel Approach to Pump Scheduling. Mathematical and Computer Modeling, L., pp-79-86, (2017)

8. Kh Fayziev, K. T Juraev ,Baytmatov Shahriddin. Fixing the slopes of the channel with combined expansion and filter seams. International Journal of dvanced Science and Technology Vol. 29, No. 8, pp. 3006-3015, (2020)

9. Mirtskhulava Ts.E. The erosion of the channels and the methodology for assessing their stability, M.: p.152, Kolos, (1987)

10. Demeulenaere A., Purwanto A., Ligout A. Design and optimization of an industrial pump: Application of genetic algorithm and neural network // Proceedings of ASME Fluid Engineering Summer Conference. Houston (USA). pp. 1519-1527, (2005)

11. Nariman-zadeh N., Amanifard N., Hajiloo A. Multiobjective Pareto optimization of centrifugal pumps using genetic algorithms // Proceedings of 11th WSEAS International Conference on Computers. pp. 123-131, Crete Island (Greece), (2007)

12. O.Glovatskiy, T. Djavburiyev, Z. Urazmukhamedova, A. Gazaryan, F.Akhmadov Interconnection of influent channel and pumping station units // XXII International Scientific Conference on Advanced in Civil Engineering / construction the formation of living environment, (2019)

13. A.Krutov, A. Azimov, S. Ruziev, A. Dumanov Modelling of turbidity distribution along channels E3S Web Conf., 97 (2019) 05046. DOI: https://doi.org/10.1051/e3sconf/20199705046.

14. A.Krutov, D. Bazarov. 
15. Experience of employment of computational models for water quality modeling E3S Web Conf., 97 (2019) 05030. DOI: https://doi.org/10.1051/e3sconf/20199705030.

16. O.R.Azizov, A.B.Saparov, Cavitation phenomenas of the running part and worker travell about blade pump // materials of the international scientific-online conference on "innovative achievements in science - 2020" Chelyabinsk-Ferghana, pp. 521-527, (2020)

17. A.Krutov, B Norkulov, D Mavlyanova Simulation of spreading of non-conservative passive substances in water bodies A Krutov et al 2020 IOP Conf. Ser.: Mater. Sci. Eng. 883012028.

18. E.Kan, M.Mukhammadiev, N.Ikramov. Methods of regulating the work of units at irrigation pumping stations. IOP Conference Series: Materials Science and Engineering, Volume 869, (2020) 042009.

19. K Dzhuraev, A Nasrulin, F Shadibekova and Sh Kurbonov Geoinformation systems at the selection of engineering infrastructure of pumped storage hydropower for the tuyamuyun complex. IOP Conference Series: Materials Science and Engineering 869 (2020) 042004 doi:10.1088/1757-899X/869/4/042004.

20. F Shaazizov, B Uralov, E Shukurov, A Nasrulin, Development of the computerized decision-making support system for the prevention and revealing of dangerous zones of flooding / E3S Web of Conferences 97, FORM-2019, 05040 (2019) 7 p. https://doi.org/10.1051/e3sconf/20199705040/.

21. Takhirjon Sultanov, Khamidkhon Fayziev, Elyor Toshmatovand Ilkhomjon Zokirov Stability of dam slopes of phosphogypsum sludge collectors. IOP Conf. Series: Materials Science and Engineering 869 (2020) 072031.

22. A.S. Ghazaryan, O.Ya. Glovatsky, B.Khamdamov, O.R. Azizov Improving the technological modes of pumping stations in the conditions of unsteady fluid movement // Scientific and practical journal "Ways to improve the efficiency of irrigated agriculture" - Novocherkassk, No. 4 (76), - pp 137-142, (2019)

23. Bazarov D., Vatin N., Obidov B., and Vokhidov O. Hydrodynamic effects of the flow on the slab of the stand in the presence of cavitation. IOP Conf. Ser. Mater. Sci. Eng. 1030, 012110 (2021).

24. Bazarov D., Markova I., Norkulov B. and Vokhidov O. Hydraulic aspects of the layout of head structures during water intake from lowland rivers. IOP Conf. Ser. Mater. Sci. Eng. 1015, 012041 (2021).

25. Bazarov D., Markova I., Sultanov S. and Kattakulov F. Dynamics of the hydraulic and alluvial regime of the lower reaches of the Amudarya after the commissioning of the Takhiatash and Tuyamuyun hydrosystems. IOP Conf. Ser. Mater. Sci. Eng. 1030, 012110 (2021).

26. Bazarov D. and Vokhidov O. Extinguishing Excess Flow Energy in Spillway Structures. In book: Proceedings of EECE 2020, LNCE 150, pp. 535-545, (2021) DOI: 10.1007/978-3-030-72404-7_52

27. Bazarov D., Markova I., Norkulov B., Isabaev K., Sapaeva M. Operational efficiency of water damless intake. IOP Conf. Ser. Mater. Sci. Eng. 869(7), 072051, (2020)

28. Obidov B., Vokhidov O., Tadjieva D., Kurbanova, U., Isakov A. Hydrodynamic effects on the flow elements of the downstream devices in the presence of cavitation. IOP Conf. Ser. Mater. Sci. Eng. 1030, 012114 (2021).

29. Krutov A., Choriev R., Norkulov B., Mavlyanova D. and Shomurodov A. Mathematical modelling of bottom deformations in the kinematic wave approximation. IOP Conf. Ser. Mater. Sci. Eng. 1030, 012147 (2021). 
30. Krutov A., Norkulov B., Uljaev F., and Jamalov F. Results of a numerical study of currents in the vicinity of a damless water intake. IOP Conf. Ser. Mater. Sci. Eng. 1030, 012121 (2021).

31. Krutov A., Norkulov B., Mavlyanova D. Simulation of spreading of non-conservative passive substances in water bodies. IOP Conf. Ser. Mater. Sci. Eng. 883(1), 012028 (2020)

32. Krutov A., Norkulov B., Nurmatov P., Mirzaev M. Applicability of zero-dimensional equations to forecast nonconservative components concentration in water bodies. IOP Conf. Ser. Mater. Sci. Eng. 883(1), 012028 (2020)

33. Krutov A., Norkulov B., Artikbekova F., Nurmatov P. Optimal location of an intake at a reservoir prone to salt diffusion. IOP Conf. Ser. Mater. Sci. Eng. 869(7), 072020, (2020)

34. Shokirov B., Norkulov B., Nishanbaev Kh., Khurazbaev M., Nazarov B. Computer simulation of channel processes. E3S Web of Conferences, 97, 05012, (2019)

35. Shomayramov, M., Norkulov B., Rakhmanov J., Tadjiyeva D., Suyunov J. Experimental researches of hydraulic vacuum breakdown devices of siphon outlets of pumping stations. E3S Web of Conferences, 97, 05009, (2019) 